\title{
Central retinal artery occlusion in Wegener's granulomatosis: a diagnostic dilemma
}

\author{
Virginia Lozano-López • Beatriz Rodríguez-Lozano • María José Losada-Castillo • \\ Esmeralda Delgado-Frías • David Dopazo-Luque • Miguel Serrano-García
}

Received: 27 June 2010 / Accepted: 7 September 2010 / Published online: 24 February 2011

(C) The Author(s) 2011. This article is published with open access at Springerlink.com

\begin{abstract}
Purpose To report a case of central retinal artery occlusion (CRAO) in a patient with biopsy-verified Wegener's granulomatosis (WG) with positive C-ANCA.

Methods A 55-year-old woman presented with a 3-day history of acute painless bilateral loss of vision; she also complained of fever and weight loss. Examination showed a CRAO in the left eye and angiographically documented choroidal ischemia in both eyes.

Results The possibility of systemic vasculitis was not kept in mind until further studies were carried out; methylprednisolone pulse therapy was then started. Renal biopsy disclosed focal and segmental necrotizing vasculitis of the medium-sized arteries, supporting the diagnosis of WG, and cyclophosphamide pulse therapy was administered with gradual improvement, but there was no visual recovery. Conclusion CRAO as presenting manifestation of WG, in the context of retinal vasculitis, is very uncommon, but we should be aware of WG in the etiology of CRAO. This report shows the difficulty of diagnosing Wegener's granulomatosis; it requires a high index of suspicion, and
\end{abstract}

\footnotetext{
V. Lozano-López $(\varangle) \cdot$ M. J. Losada-Castillo · M. Serrano-García Department of Ophthalmology,

Hospital Universitario de Canarias,

Ctra. Gral del sur s/n,

38300 La Laguna, Tenerife, Spain

e-mail: vlozano77@hotmail.com

B. Rodríguez-Lozano $\cdot$ E. Delgado-Frías

Servicio de Reumatología, Hospital Universitario de Canarias, Ctra. Gral del sur s/n,

38300 La Laguna, Tenerife, Spain

D. Dopazo-Luque

Department of Radiology, Hospital Universitario de Canarias,

Ctra. Gral del sur s/n,

38300 La Laguna, Tenerife, Spain
}

we should obtain an accurate medical history and repeat serological and histopathological examinations. It emphasizes that inflammation of arteries leads to irreversible retinal infarction, and visual loss may occur

Keywords Central retinal artery occlusion · Kidney failure · Retinal vasculitis · Wegener's granulomatosis

\section{Introduction}

Signs of central retinal artery occlusion (CRAO) often herald associated systemic vascular events. There are a number of ways in which the retinal arteries can become blocked; the most common is by an embolus. Alternatively, there may be a sudden narrowing of the vessels due to atherosclerotic changes, vasculitis, vascular spasm, circulatory collapse, dissecting aneurysm, and hypertensive arterial necrosis [1]. Early recognition and treatment may preserve eye function. A detailed analysis of disease associated with the acute visual loss is necessary to elucidate the cause [2].

We report a case of CRAO being the first manifestation of Wegener's granulomatosis (WG) developed in the setting of retinal vasculitis. Besides, renal failure with cantineutrophil cytoplasmic antibodies (C-ANCA) and medium-vessel necrotizing inflammation shown by the biopsy. Retinal involvement is relatively uncommon, occurring in approximately $5-12 \%$ of patients. Retinal vascular manifestations range from relatively benign cotton-wool spots, with or without associated intraretinal hemorrhages, to more severe vaso-occlusive disease, including CRAO [3]. WG is a rare, chronic multisystemic disease, with an annual incidence of ten cases per million population $[4,5]$. More common in Caucasians with a peak 
Fig. 1 a Fundus photograph of the right eye shows a slightly pale retina in the posterior pole. b Fundus photograph of the left eye shows obstruction of the central retinal artery with yellowish retinal appearance and characteristic cherry-red spot at the foveola
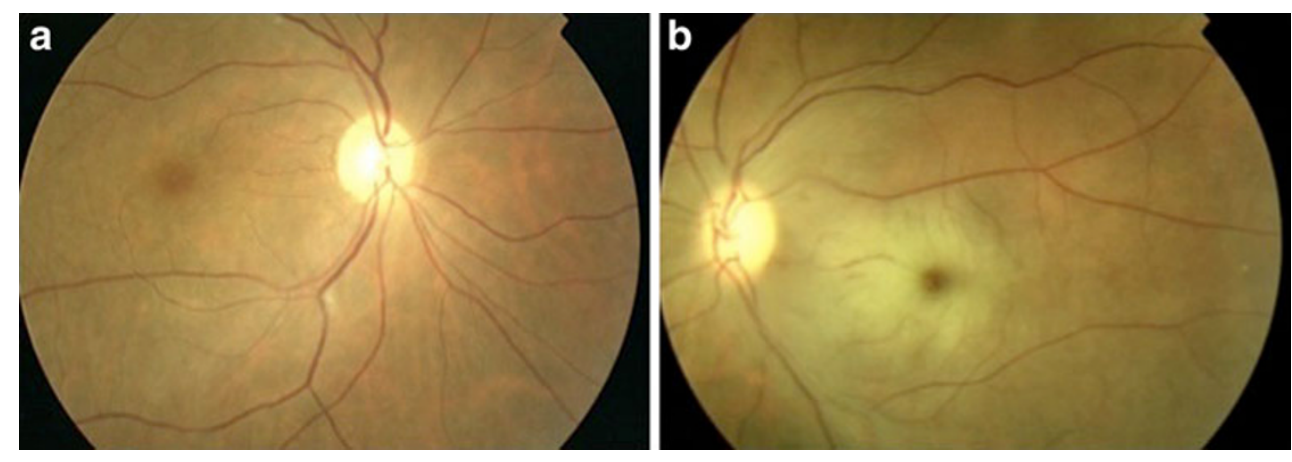

incidence in the fifth decade of life [6], WG has a predilection for affecting the upper and lower respiratory tracts and kidneys [1, 7] and the eyes; central nervous system can also be affected [8].

The purpose of this report is to consider $\mathrm{WG}$ in the differential diagnosis of CRAO, although this entity is rarely encountered as presenting sign of the disease.

\section{Case report}

A 55-year-old woman was admitted to our department with complaints of progressive vision loss in the left eye over the course of 3 days. The patient reported weight loss, asthenia, and polyarthralgia with progressive weakness persisting for 2 months. Four weeks earlier, she was referred to the local hospital because she experienced persistent fever and increasing shortness of breath that had not responded to amoxicillin-clavulanate treatment; she was found to have pneumonia and was empirically treated with various antibiotics. She also developed an episode of sudden onset, painless reduced vision in the right eye after admission. She had presented to the emergency department 2 months ago with complaints of fever and cough with mucopurulent expectoration, and she had been treated with amoxicillin-clavulanate.

The patient was referred to our Ophthalmology Department by the rheumatologist who provided her with further management. Entering examination revealed no perception of light in her left eye while the visual acuity (VA) of the right eye was 20/60. Anterior segment appeared unremarkable. Intraocular pressure was normal. Dilated fundoscopy of the left eye demonstrated a cherry-red spot at the macula with edematous retina, characteristic of CRAO (Fig. 1a). The right fundus showed a slightly pale retina in the posterior pole (Fig. 1b). The patient was not able to have fluorescein angiography and indocyanine green angiography due to her bad general conditions. On physical examination, she was febrile and short of breath; her blood pressure was also high. Her cardiopulmonary examination revealed increased sounds at the right lung base. In her musculoskeletal examination, there was swelling and sensitivity to palpation in the proximal interphalangeal joints of the right hand and also upon her shoulders, left ankle, and knee. Her past medical history included hypertension and chronic venous insufficiency. The initial patient workup included complete blood count (showing anemia, neutrophilic leukocytosis, and thrombocytosis); biochemistry results showed an erythrocyte sedimentation rate of $5 \mathrm{~mm}$ in the first hour and a CRP higher than $90 \mathrm{mg}$ / 1 , a blood urea nitrogen level of $28 \mathrm{mg} / \mathrm{dL}$, and a creatinine level of $2.5 \mathrm{mg} / \mathrm{dL}$, but normal electrolytes and liver function. Urinalysis revealed moderate hematuria and mild pyuria. Chest X-ray was negative for pneumonia (Fig. 2). Possibility of systemic vasculitis was kept in mind, and intravenous methyprednisolone pulses were started (three pulses of $0.5 \mathrm{~g}$ each). Fluorescein angiography taken a few days later disclosed delayed retinal arterial filling in both eyes and nonperfusion through affected cilioretinal artery in the left eye and, surprisingly, affected choroidal blood flow with choroidal filling defect in the posterior pole of the right eye (Fig. 3a) and in peripheral areas of the left eye (Fig. 3b), rarely seen in patients with CRAO [9].

Additional blood tests revealed anti-nuclear antibody titer of 1:40, anti-SSA/Ro-antibody-positive, negative hepatitis and HIV antibodies, and also progressively increasing serum creatinine levels with a creatinine clearance of $18 \mathrm{~mL} / \mathrm{min}$. Therefore, the patient developed renal failure. The diagnosis of WG was supported by strongly positive $\mathrm{C}$ -

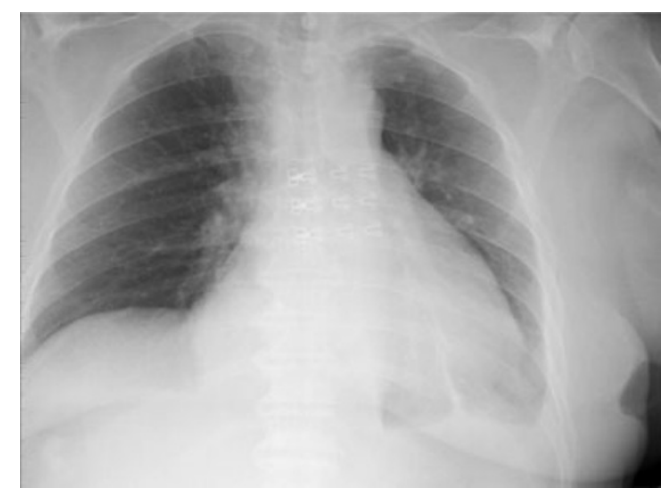

Fig. 2 Chest X-ray revealing enlarged heart, but neither pleural nor parenchymal abnormalities 
Fig. 3 a Fuorescein angiography of the right eye revealing hypoperfusion of the occluded choroid in the posterior fundus and staining of the optic disc in both eyes. b Fuorescein angiography of the left eye revealing nonperfusion through affected cilioretinal artery and segmental filling defect of the peripheral choroid and staining of the optic disc in both eyes
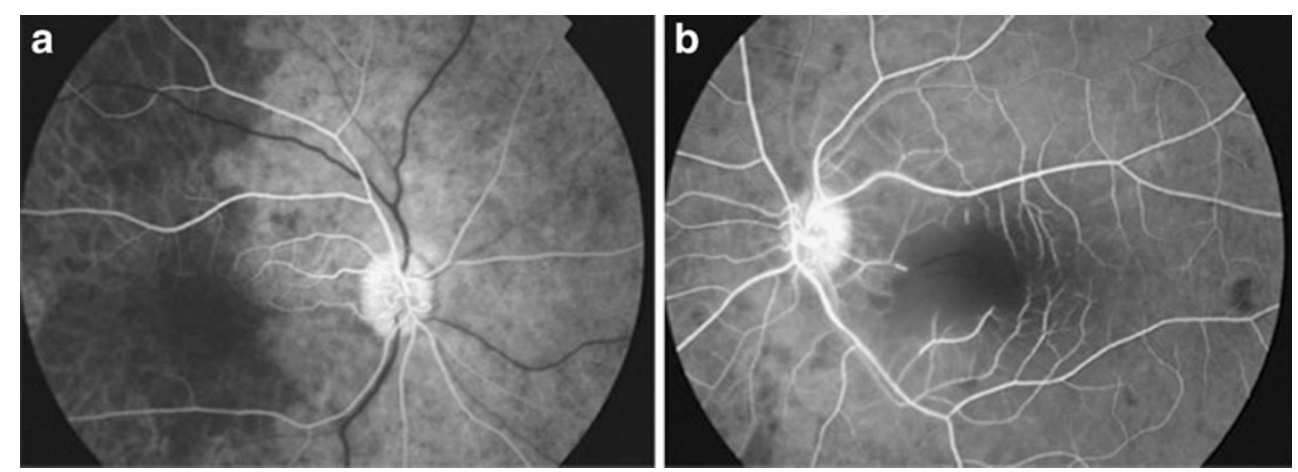

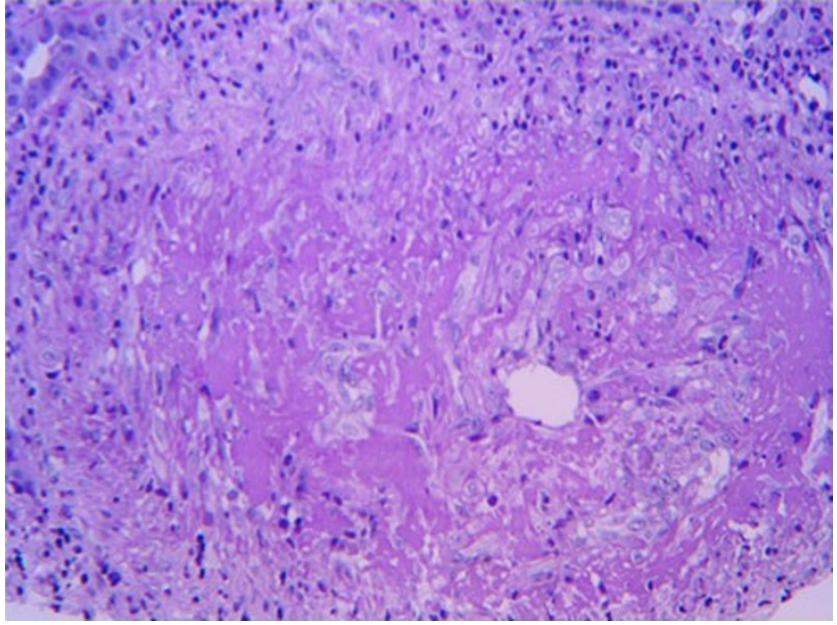

Fig. 4 Kidney biopsy showing a vasculitic process of medium-sized arteries with central necrosis and surrounding histiocytes
ANCA anti-PR3 antibodies confirmed by kidney biopsy that showed focal and segmental necrotizing vasculitis of the medium-sized arteries (Fig. 4). Computed tomography (CT) of the brain and orbits was normal. CT scan of the chest, abdomen, and pelvis revealed pleural effusion, perirenal hematoma, and splenic infarction (Fig. 5a, b). The patient was aggressively treated with cyclophosphamide for six consecutive cycles, which resulted in a total cumulative dose of $6.8 \mathrm{~g}$. Concomitant administration of corticosteroid (1 mg/kg/day oral prednisone) was used, and the patient gradually improved with radiographic clearing of pulmonary abnormalities and normalization of laboratory studies. To maintain remission, oral azathioprine $(2 \mathrm{mg} / \mathrm{kg} /$ day) and prednisone $(15 \mathrm{mg}$ /day) were administered and were then gradually tapered. Two years after diagnosis, VA remains 20/60 in the right eye with no light perception in the left eye. The funduscopy shows bilateral optic disc pallor (Fig. 6a, b). Since then, the patient has been clinically stable, as seen in the OCT (Fig. 7a, b).

\section{Discussion}

What initially appeared to be CRAO was, in fact, an uncommon manifestation of retinal vasculitis due to WG.
Fig. 5 a $\mathrm{CT}$ scan of the upper abdomen at the time of the biopsy shows pleural effusion and perirenal hematoma (arrow). b CT scan of the upper abdomen shows homogeneous hypodensity in the spleen (arrow)
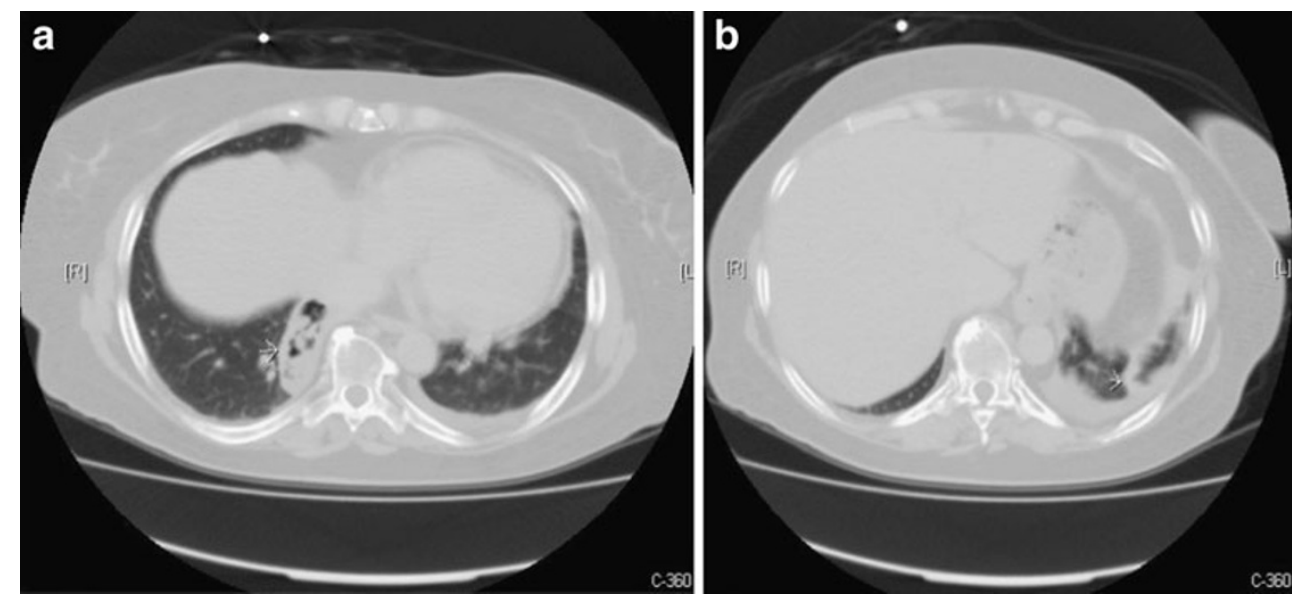

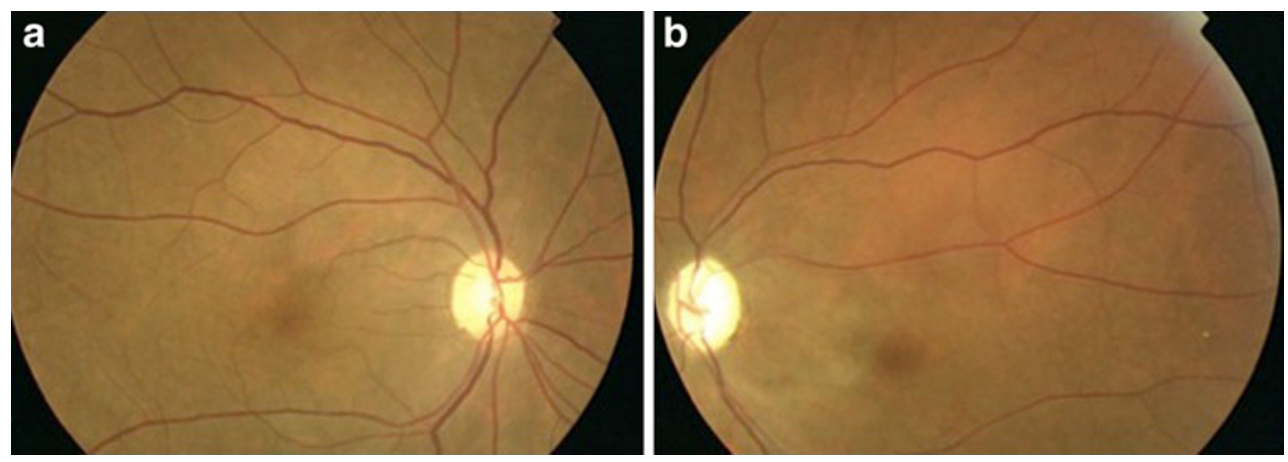

Fig. 6 a Right eye fundus showing a mild pallor of the optic disc. b Left eye fundus showing a marked pallor of the optic disc

Our patient had CRAO in the left eye as well as bilateral choroidal ischemia. Iida, Kinyoun, and Mirza have reported respective cases of $\mathrm{WG}$ associated with bilateral choroidal infarctions [10]. We should consider WG in the differential diagnosis of patients presenting with CRAO and retinal vasculitis. Although much progress has been made in retinal vascular occlusion syndromes, more understanding is still needed to prevent the sequelae of these blinding disorders [11]. The mechanism underlying retinal vasculitis in WG remains unknown; the pathogenesis can be inferred by vasculitis mediated, in part, by stimulatory autoantibodies (ANCA). This process causes the neutrophils to bind to endothelium and to release mediators that injure the vessel wall $[7,12]$.

\section{a}
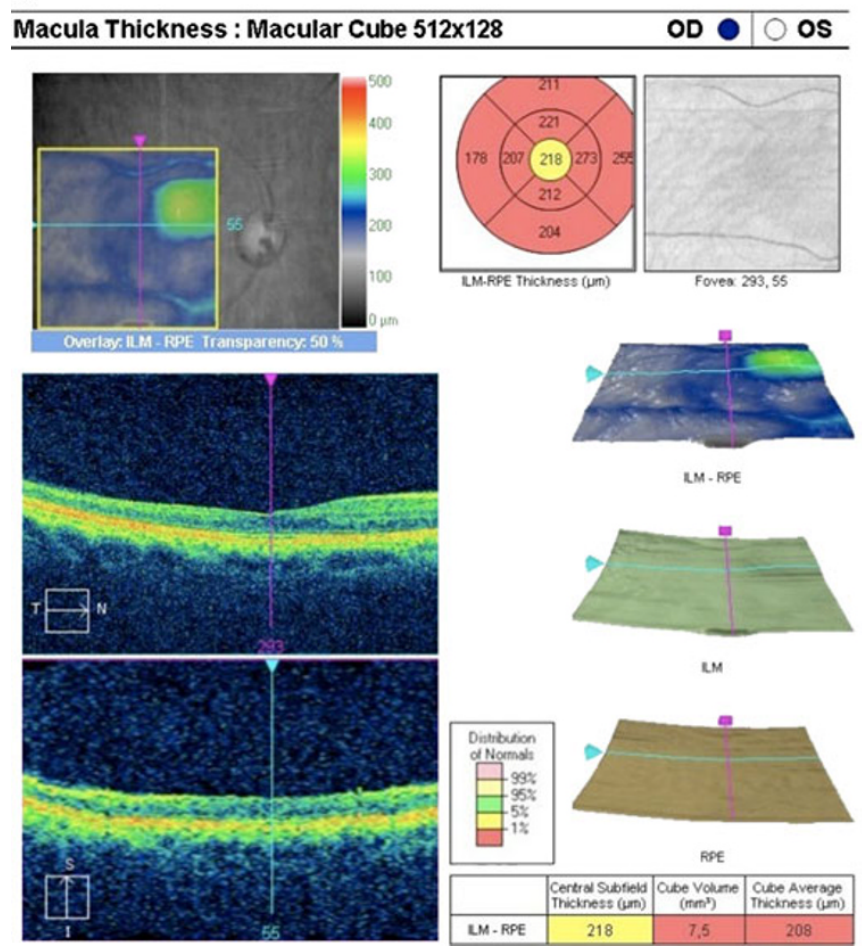

IM. RPE

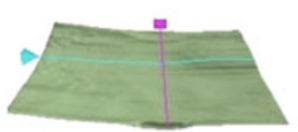

in

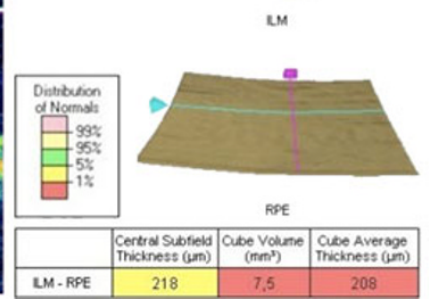

WG has rarely been implicated in CRAO, although more commonly in retinal vasculitis. Ocular manifestations may represent the first sign of $\mathrm{WG}$, and early diagnosis could prevent potentially life-threatening renal failure with its increased morbidity and mortality $[13,14]$. In the acute setting, diagnosis is usually clinical and should include patient's medical history, review of systems, and ocular examination. Therefore, a multi-disciplinary approach is required [15]. Laboratory investigations should be directed towards suspected diagnosis [16]. Fluorescein angiography may be helpful and frequently shows that the vasculitis is more extensive than the clinical examination suggests [17]. An angiographic study showed that retinal vasculitis and choroidal inflammation develop independently from each

\section{b}

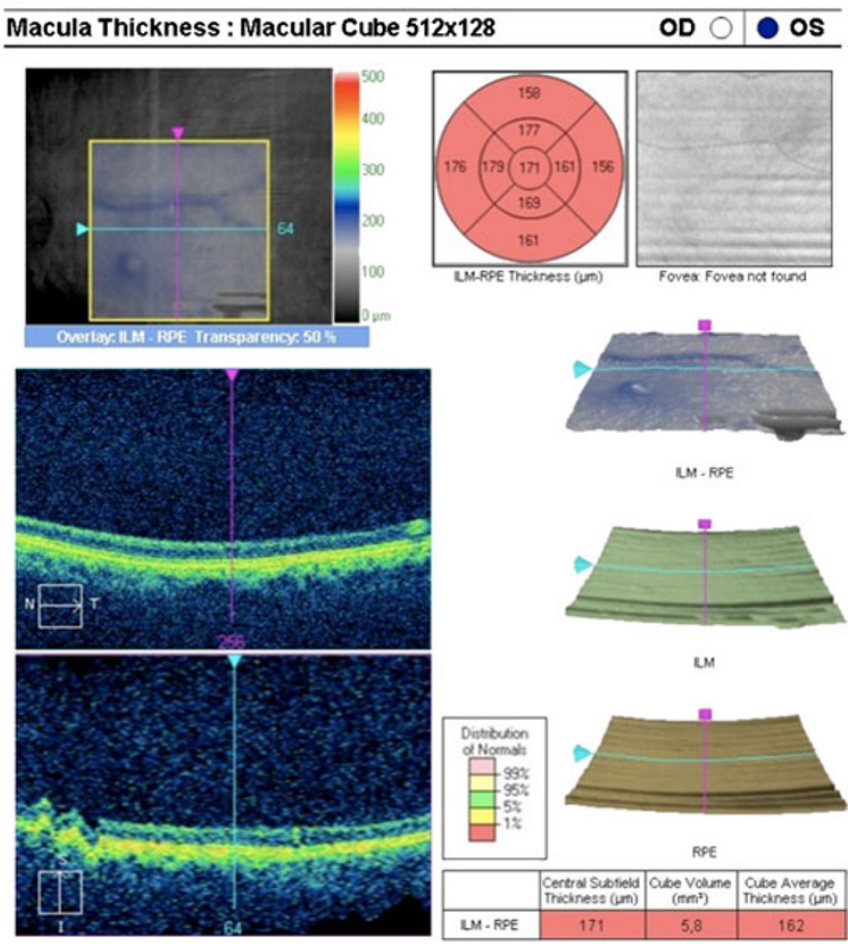

Fig. 7 a The OCT of the right eye at final examination shows no macular edema. b The OCT of the left eye at final examination shows no macular edema 
other [9]. Treatment regimen often includes prednisone and cyclophosphamide [18].

WG is a rare entity that can affect almost any part of the body [6]. In fact, WG is diagnosed by the presence of two of these four criteria established by the American College of Rheumatology, which includes nasal or oral inflammation, abnormal chest radiograph, excessive urinary sediment, and granulomatous inflammation on biopsy [7]. In WG, $80-95 \%$ of cases are C-ANCA (anti-PR3)-positive [4].

Our case emphasizes that serious visual complications may occur in WG, and early diagnosis is necessary because delay in diagnosis is unfortunately common and this often leads to a worse prognosis $[4,5]$.

Acknowledgements The authors are grateful to Dr. Corviniano Rodríguez for his valuable interest in uveitis and who has provided us very interesting cases.

Financial disclosure(s) The author(s) have no proprietary or commercial interest in any materials discussed in this article.

Open Access This article is distributed under the terms of the Creative Commons Attribution Noncommercial License which permits any noncommercial use, distribution, and reproduction in any medium, provided the original author(s) and source are credited.

\section{References}

1. Timoney PJ, Pate JC, Pearson PA, Crandall J (2009) Bilateral CRAO in a patient with acute pancreatitis. Retin Cases Brief Reports 3:308-309

2. Chen YJ, Chen WL, Tsao YT (2009) Sentinel central retinal artery occlusion: a forme fruste of idiopathic renal infarction. Am J Emerg Med 27:253

3. Bullen CL, Liesegang TC, McDonald TJ, DeRemee RA (1983) Ocular complications of Wegener's granulomatosis. Ophthalmology 90:279-290
4. Costello F, Gilberg S, Karsh J, Burns B, Leonard B (2005) Bilateral simultaneous central retinal artery occlusions in Wegener granulomatosis. J Neuro-Ophthalmol 25:29-32

5. Khan AM, Elahi F, Hashmi SR, Mahida KH, Ingrams DR (2006) Wegener's granulomatosis: a rare, chronic and multisystem disease. Surgeon 4(1):45-52

6. Pakrou N, Selva D, Leibovitch I (2006) Wegener's granulomatosis: ophthalmic manifestations and management. Semin Arthritis Rheum 35:284-292

7. Kelley JM, Edberg JC, Kimberly RP (2010) Wegener's granulomatosis: a model of auto-antibodies in mucosal autoimmunity. Clin Immunol 134:104-112

8. Blaise P, Robe-Collignon N, Andris C, Rakic JM (2007) Wegener's granulomatosis and posterior ischemic optic neuropathy: atypical associated conditions. Eur J Intern Med 18:326327

9. Herbort CP, Cimino L, El Asrar AM (2004) Ocular vasculitis: a multidisciplinary approach. Curr Opin Rheumatol 17:25-55

10. Iida T, Spaide RF, Kantor J (2002) Retinal and choroidal arterial occlusion in Wegener's granulomatosis. Am J Ophthalmol 133:151-152

11. Haymore JG, Mejico LJ (2009) Retinal vascular occlusion syndromes. Int Ophthalmol Clin 49(3):63-79

12. Kallenberg CG, Brouwer E, Mulder AH, Stegeman CA, Weening JJ, Tervaert JW (1995) ANCA pathophysiology revisited. Clin Exp Immunol 100:1-3

13. Harper SL, Letko E, Samson CM, Zafirakis P, Sangwan V, Nguyen Q, Uy H, Baltatzis S, Foster CS (2001) Wegener's granulomatosis: the relationship between ocular ans systemic disease. J Rheumatol 28:1025-1032

14. Choopong P, Tauber J, Foster CS (2008) Wegener granulomatosis. Contemp Ophthalmol 7:191

15. Tsuzuki K, Fukazawa K, Takebayashi H, Hashimoto K, Sakagami M (2009) Difficulty of diagnosing Wegener's granulomatosis in the head and neck region. Auris Nasus Larynx 36:64-70

16. Perez VL, Chavala SH, Ahmed M, Chu D, Zafirakis P, Baltatzis S et al (2004) Ocular manifestations and concepts of systemic vasculitides. Surv Ophthalmol 49:399-418

17. Bisighini SL, Pagliuso LM (1997) Retinal vasculitis: a diagnostic dilemma. Clin Eye Vis Care 9:71-84

18. Levi M, Kodsi SR, Rubin SE, Lyons C, Golden R, Olitsky SE, Christiansen S, Alcorn DM (2008) Ocular involvement as the initial manifestation of Wegener's granulomatosis in children. J AAPOS 12(1):94-96 